\title{
ELECTRON HEAT TRANSPORT IN A TOKAMAK WITH DESTROYED MAGNETIC SURFACES
}

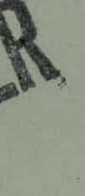

BY

A. B, RECHESTER

AND

M. N, ROSENBLUTH

\section{PLASMA PHYSICS LABORATORY}

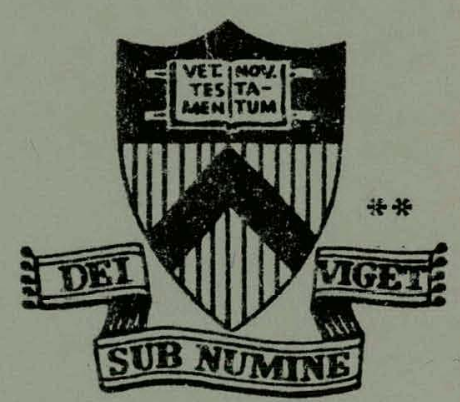

\section{PRINCETON \\ UNIVERSITY PRINCETON, NEW JERSEY}

This work was supported by U. S. Energy Research and Development Administration Contract EY-76-C-02-3073. Reproduction, translation, publication, use and disposal, in whole or in part, by or for the United States Government is permitted. 


\section{DISCLAIMER}

This report was prepared as an account of work sponsored by an agency of the United States Government. Neither the United States Government nor any agency Thereof, nor any of their employees, makes any warranty, express or implied, or assumes any legal liability or responsibility for the accuracy, completeness, or usefulness of any information, apparatus, product, or process disclosed, or represents that its use would not infringe privately owned rights. Reference herein to any specific commercial product, process, or service by trade name, trademark, manufacturer, or otherwise does not necessarily constitute or imply its endorsement, recommendation, or favoring by the United States Government or any agency thereof. The views and opinions of authors expressed herein do not necessarily state or reflect those of the United States Government or any agency thereof. 


\section{DISCLAIMER}

Portions of this document may be illegible in electronic image products. Images are produced from the best available original document. 
NOTICE

This report was prepared as an account of work sponsored by the United States Government. Neither the united States nor the United States Energy Research and Development Administration, nor any of their employees, nor any of their contractors, subcontractors, or their employees, makes any warranty, express or implied, or assumes any legal liability or responsibility for the accuracy, completeness or usefulness of any information, apparatus, product or process disclosed, or represents that its use would not infringe privately owned rights.

Printed in the United States of America.

Available from

National Technical Information Service

U. S. Department of Commerce 5285 Port Royal Road Springfield, Virginia 22151

Price: Printed Copy $\${ }_{*}$; Microfiche $\$ 3.00$

*Pages

$1-50$

$51-150$

$151-325$

$326-500$

$501-1000$
NTIS

Selling Price

$\$ 4.00$

5.45

7.60

10.60

13.60 


\section{ELECTRON HEAT TRANSPORT IN A TOKAMAK \\ WITH DESTROYED MAGNETIC SURFACES}

by

A. B. Rechester

Princeton University, Plasma Physics Laboratory

Princeton, ivew Jersey 08540

and

M. N. Rosenbluth

Institute for Advanced Study

Princeton, New Jersey 08540

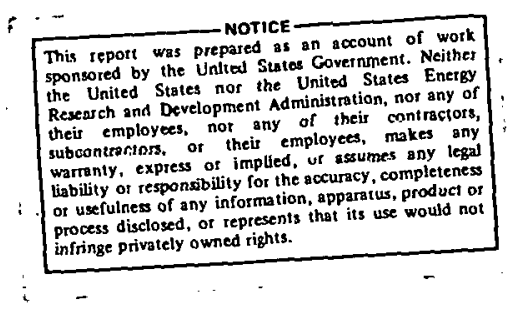

PPPL-1386

October 1977 
ELECTRON HEAT TRANSPORT IN A TOKAMAK WITH DESTROYED

MAGNETIC SURFACES *

A. B. Rechester

Plasma Physics Laboratory, Princeton University, Princeton, New Jersey 08540

and

M. N. Rosenbluth

Institute for Advanced Study, Princeton, New Jersey 08540

\section{ABSTRACT}

Formulas for the electron thermal conductivity have been derived in the collisional and collisionless limits for the case of destroyed magnetic surfaces. 
The aim of this article is to consider electron heat transport due to parallel thermal conductivity in a tokamak with destroyed magnetic surfaces. There is evidence of runaway electron leakage ${ }^{2}$ from tokamaks which indicates that such magnetic braiding ${ }^{3}$ may exist. Moreover, recent microinstability theories ${ }^{4}$ predict the formation of magnetic perturbations with dimensions $r_{i}$ (ion gyro-radius) or slightly less.

First we review the most important geometric characteristics of the braided magnetic field. We use as an example a magnetic configuration in cylindrical geometry, $\vec{B}=B_{z} \vec{z}+B_{\theta}(r) \hat{\theta}+\delta \vec{B}$, but most concepts can be easily generalized to toroidal configurations. In order to model toroidal periodicity, we assume that the system is periodic in the z-direction with period $2 \pi R$. Then $\delta \overrightarrow{\mathrm{B}}$ can be written in the form

$$
\delta \vec{B}=\sum_{m, n} \cdot \vec{b}_{m n}(r) \exp i(m \theta-n z / R)+c \cdot c
$$

If there is only one harmonic in $\mathrm{Eq} .(1)$; then the field is helically symmetric and has exact magnetic surfaces. These surfaces have the shape of so-called magnetic islands near the rational cylindrical surface $r_{m n}$, defined by the condition $q(r)=r B_{z} / R B_{\theta}=l / i(r)=m / n$. The width of the separatrix of the island is given by the formula, 5

$$
\Delta_{m n}=\left.4 \sqrt{2 \frac{R}{m}|| b_{m n r}(r) \mid / B_{z}(d \bar{l} / d r)}\right|_{r}=r_{m n}
$$

In the case of many harmonics being present, mapping is used as a. practical way to determine if magnetic surfaces exist or not. 6 
Surfaces exist if the points of intersection. of a field line with planes $z=2 \pi n R$ lie on a smooth closed curve. If the mapping is not a smooth curve but rather a sequence of irregular points which can fill the whole area, then we may say that magnetic surfaces are destroyed. The transition between these two cases is quite sharp and is described by a stochasticity parameter, 7 $s=1 / 2\left(\Delta_{m n}+\Delta_{m^{\prime} n^{\prime}}\right) /\left|r_{m n}-r_{m^{\prime} n^{\prime}}\right|$, where $m, n$ and $m^{\prime}, n^{\prime}$ represent any two harmonics which have neighboring rational surfaces. If $s \geq 1$, then magnetic surfaces are destroyed in the region between $r_{\text {Hu }}$ and $r_{m} n^{\prime}$. तnd the field linco wonder eryulically. $s=1$ corresponds to overlapping of islands of different helicity. ${ }^{9}$ The transition region is very complicated ${ }^{7}$ and we will be discussing mainly the case of well destroyed surfaces, $\mathbf{s} \gg 1$, with dense rational surfaces.

Consider à small circle nf radius ${ }^{\circ} 0$ in the plane $z=$ const and map it by solving the equation $d r / d z=B_{1} / B_{z}$, $r d \theta / d z=B_{\theta} / B_{z}$. This magnetic mapping is area preserving, as a consequence of the equation $\operatorname{div} B=0$.

There will be two different stages of evolution of our area. First, it will move as a whole and also it will deform its shape stretching in one direction and contracting in the other. This process can be described analytically for continuous mapping by the equation.

$$
\ell(z)=\ell_{0} \exp \left(z / L_{c}\right)
$$

Here ${ }^{7} L_{c}=\pi R / \ln (\pi s / 2)$. Such behavior is called stochastic instability of trajectories. The width $\delta$ of the area will exponentially decrease in order to conserve the total area, i.e., 
$\delta(z)=\ell_{0} \exp \left(-z / L_{c}\right)$. When $\ell(z) \geq r / m$, where $m$ is a characteristic mode number, different parts of our stretched area start to move almost independently. The distance $I_{c 0}=I_{c} \ell n\left(r / m l_{0}\right)$ plays the role of a correlation length for the area. At this stage of the evolution the area looks very complicated, as depicted in Fig. 1. It can be shown that the average squared radial displacement of the area can be described by a diffusion formula

$$
\left.<(\Delta r)^{2}\right\rangle=2 L D_{s t}
$$

$I$ is the distance in the $z$ direction, $L \gg L_{c o}$ and $D_{s t}$ is given by the quasilinear formula: ${ }^{1}$

$$
D_{s t}(r)=\pi R \sum_{m, n}\left|b_{m n r}(r)\right|^{2} / B_{z}^{2} \cdot \delta\left(\frac{m}{q(r)}-n\right) .
$$

All processes of mapping are strictly reversible, but because the width of the area becomes extraordinarily small, any small spreading because of motion perpendicular to the field lines can be of great importance as we will see later. The precise mathematics and all details can be found in a number of good reviewe on the eubject. 7,8

Let us turn now to the subject of this letter: electron heat transport in a braided magnetic field. The coefficient of thermal conductivity $x$, is proportional to the coefficient of self diffusion $D$. Suppose that in some small region we mark instantaneously a number of electrons and then watch for the time evolution of their radial distribution. If the radial spreading of test electrons is a diffusion process, then $D=$ $\langle(\Delta r)\rangle^{2} / 2 t$, where $\left\langle(\Delta r)^{2}\right\rangle$, is the mean square of the radial 
displacements of electrons during the time interval $t$. We will consider two limits: collisionless, when the mean free path $\lambda \gg I_{C}$ and collisional, $\lambda \ll L_{C}$. [We will make a number of simplifying assumptions and not attempt to give detailed numerical coefficients.]

Consider the collisionless case. We assume that guiding center trajectories coincide with the field lines. Instead of considering many discrete particles, we visualize just one "particle" which is spread over some initial area of the dimension $r_{e}$ (electron gyro-radius) with equal probability. Then parallel motion along the field lines produces a continuous mapping of the area. We may treat collisions as a discrete process which take place periodically with the time interval $\tau$. As a result of collisions, the parallel velocity of the particle will change its direction or remain the same with equal probability. Also the whole area will instantaneously diffuse radially a distance $\mathfrak{r}_{e}$ (or an electron "banana" width in the toroidal case). The latter process models the perpendicular jump of the guiding center at the time of collision.

Let us start our experiment. Initially, we have a small circle of radius $r_{e}$. It moves a distance $\lambda$ along the trajectory, mapping into a complicated thin region of the kind drawn in Fig. $l(c)$, with the width $\delta \simeq r_{e} \exp \left(-\lambda / L_{c}\right):$ The average squared displacement of its elements in a radial direction is equal to $\left\langle(\Delta I\rangle^{2}\right\rangle=2 \mathrm{D}_{s t^{\lambda}}$. 'Ihe collision now increases the width of this area to $r_{e}$. We can cut now our area into a large number of small square pieces of the size $r_{e}$ and proceed in exactly the same way as in the first step, see Fig. I(c). 
Because for the collisionless case $r_{e}>\delta\left(\lambda_{i}\right)$, each of these new elements will evolve on the second step almost independently from its previous history. Obviously, the spreading of our area in the radial direction is similar to a random walk with the diffusion coefficient given by

$$
D=\left\langle(\Delta r)^{2}\right\rangle / 2 \tau=D_{s t} v \text {. }
$$

This formula does not depend on collision frequency in spite of the importance of collisional spreading.

It is instructive to compare this case with the similar evolution without any perpendicular motion. Suppose that the particle reverses its parallel velocity after the first collision. Then the second step area will map exactly back to the initial small circle. Obviously, the only way for it to expand in the radial direction is to diffuse collisionally along the field line. During a time $t \gg \tau$, the average squared distance moved by a particle in the $z$ direction is $L^{2}=x_{11} t$. This gives $\left\langle(\Delta r)^{2}>=D_{s t}\left(x_{n} t\right)^{1 / 2}\right.$, which is $(t / \tau)^{1 / 2}$ times smaller than the diffusion given by Eq. (6).

Consider now the collisional case $\lambda \ll I_{C}$. Any substantial spreading of the area in the radial direction due to the mapping will take place after the particle has collided many times to move a distance. $L \geqslant L_{c}$. Because of this, we will consider perpendicular motion as a continuous process with the diffusion coefficient $x_{\perp}$. The parallel motion and the effect of collisions on it will be treated the same way as before. Consider a small element of evolving area which has a typical size $\delta$. There will be two competing processes: due to stochastic instability, the width of this element will decrease 
exponentially: $d \delta / d L=-\delta / L_{C}$, where $d L$ is the distance which the particle moves during the time $d t$. For any $d L>\lambda$, the particle is diffusing in the $z$ direction so that $d t \simeq(d L)^{2} / x_{1}$. During the same time dt this element will increase its width because of perpendicular diffusion $\mathrm{d} \delta \simeq\left(x_{1} \mathrm{~d} t\right)^{1 / 2}=\mathrm{dL}\left(\mathrm{x}_{11} / x_{\perp}\right)^{1 / 2}$. The balance between these two processes allow us to estimate that $\delta=\mathrm{L}_{\mathrm{c}}\left(\mathrm{x}_{\perp} / \mathrm{x}_{11}\right)^{1 / 2}$. We assume here that $\delta \ll \mathrm{r} / \mathrm{m}$. If we cut the area into small pieces of size $\delta$ then the correlation length. for them may be calculated by setting $\ell_{0}=\delta$ and $\ell(z)=r / m$ in Eq. (3), to find:

$$
L_{c \delta}=L_{c} \ln \left(\frac{r}{m L_{c}} \sqrt{\chi_{11} / x_{\perp}}\right)
$$

with the corresponding time $t_{\delta} \sim I_{c \delta}^{2} x_{11}$. Thus, $t_{\delta}$ is the time during which a particle may be thought of as orbiting along a single field line before diffusing to a new field line whose trajectory is no longer correlated with the original one. This complicated continous evolution can be considered simply as a random walk with the step size $\left\langle(\Delta r)^{2}\right\rangle=D_{s t} L_{c} \delta$. This gives us for the diffusion coefficient,

$$
D=D_{s t} X / L_{c \delta}
$$

Diffusion is reduced by a factor of $\lambda / L_{c \delta}$ from the collisionless case, Eq. (6).

Now that we have calculated the relevant transport coefficients in a braided magnetic field, a logical step in understanding would be to assess the effects of the stochasticity on the nonlinear behaviour of the microinstabilities which are alleged to be its cause. 4 This is obviously a formidable task. 
A more realistic approach might be to assess the effects of the thermal conductivity we have estimated, and related transport coefficients such as electron viscosity, on the linear theory of those modes--estimating saturation of stochasticity to occur at the marginal stability point. Out of such a detailed study might come an explanation of confinement scaling.

In this paper, however, we restrict ourselves to a much more limted objective, merely examining some implications of the assumption that observed energy losses are due to magnetic braiding. We stress that we have not ruled out alternative explanations of anomalous losses.

A possible difficulty in applying our model to present tokamaks comes from consideration of the confinement of energetic runaway electrons. While not very well known, their confinement time is measured to be about equal to or slightly longer than that for thermal electrons. From Eq. (6), we would infer however that their confinement time should be about 15 times smaller. One possible explanation for this anomaly is that the gyroradii of such particles is fairly large, comparable to ion gyroradii, so that their drift orbits are not affected by fine scale braiding. This would argue that the predominant scale for braiding is somewhat smaller than an ion gyroradius, consistent with microinstability theory and turbulent density fluctuation measurements. We may use Eq. (6) to infer the diffusion coeficient. Observed confinement times are crudely fit by $\tau=10^{-19} \mathrm{n}^{2}$ (c.g. s units) from which we infer, at a density $n=10^{14}$, that the experimental diffusion coefflclent $D=10^{4} \mathrm{~cm}^{2} / \mathrm{sec}$ and hence, 
$\mathrm{D}_{\mathrm{st}}=10^{-5} \mathrm{~cm}$. From Eq. (2), we see that $\mathrm{D}_{\mathrm{st}} \approx \mathrm{R}\left|\mathrm{b}_{\mathrm{m}, \mathrm{n}}\right|^{2} \mathrm{~m}_{\max } / \mathrm{B}_{z}^{2} \approx$ $R\left|B_{s t}\right|^{2} / B_{z}^{2}$. Here we have assumed, as is reasonable for microinstabilities, that all modes $m, n$ have roughly equal amplitudes for mode number $m<m_{\max }$ (where $\mathrm{m}_{\max }$ is to be determined from the microinstability theory). We have also assumed a radial extent of the mode comparable to its wavelength. Equating the experimental and theoretical values, we see that $\left|B_{s t}\right|^{2} / B_{z}^{2} \sim 10^{-8}$ is adequate to give the observed losses if the field is indeed stochastic. As we have riscussed earlicr, the condition for this is roughly that islands should overlap. The mean distance between adjacent rational surfaces is approximately $\mathrm{r} / \mathrm{m}_{\max }^{2}$ and uṣing Eq. (2), we obtain the approximate condition for stochasticity $\left|\mathrm{B}_{\mathrm{st}}\right| / \mathrm{B}_{z}>0.1 / \mathrm{m}_{\max }^{5 / 2} \cdot$ current microinstability theories and fluctuation measurements indirate. that $m_{\max } \geq r / x_{i}$. In typical tokamak experiments $r / r_{j}>10^{2}$ and the condition for stochasticity is well satisfied. We conclude then that if the perturbed magnetic fields are indeed caused by microinstabilities on the scale of an ion gyroradius, then fields of an amplitude sufficient to explain by stochastic diffusion the observed energy losses would also be sufficient to produce stochasticity--thus making the picture self-consistent in this respect. There remains of course the upen question whether this transport can explain the saturation of the microinstabilities at the observed level.

It is a pleasure to acknowledge discussions with T. H. Stix. Related considerations will be published by him in a forthcoming paper in Nuclear Fusion. 


\section{REFERENCES}

Work supported by U. S. Energy Research and Development Administration Contract No. $E(11-1)-3073$ and $E(11-1)-3237$.

I M. N. Rosenbluth, R. Z. Sagdeev, J. B. Taylor and G. M. Zaslavsky, Nucl. Fusion 6,297 (1966) and N. N. Filonenko,

R. Z. Sagdeev and G. M. Zaslavsky, Nucl. Fusion 7, 253 (1967).

2 J. D. Strachan, Nucl. Fusion 165, 743 (1976).

3 T. H. Stix, Phys. Rev. Lett. 30,833 (1973).

4 J. F. Drake and Y. C. Lee, Phys. Rev. Lett 39, 453 (1977),

I. Chen, P. H. Rutherford and W. M. Tang, Phys. Rev. Lett. 39, 460, (1977) and J. D. Callen, submitted to Phys. Rev. Lett. (1977).

5 A. B. Rechester and T. H. Stix, Phys. Rev. Lett. 36, 587 (1976) .

6 L. Spitzer, Phys. Fluias I, 253 (1958).

7 G. M. Zaslavsky and B. V. Chirikov, Soviet Physics Uspekhi 14, 543, (1972).

8 R. Balescu, Equilibrium and Nonequilibrium Statistical Mechanics (Wiley-Interscience, New York, 1975) 695.

9 We should mention here that for the island overlapping condition

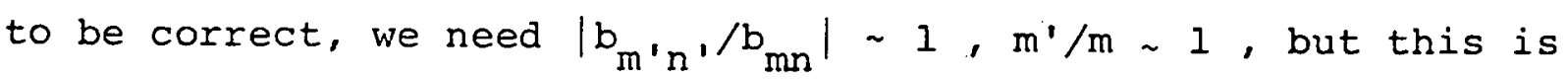
adequate for our purposes. See A. B. 'Rechester paper G 14 , presented at the Sherwood Theory Meeting, San Diego, CA., 1977. 


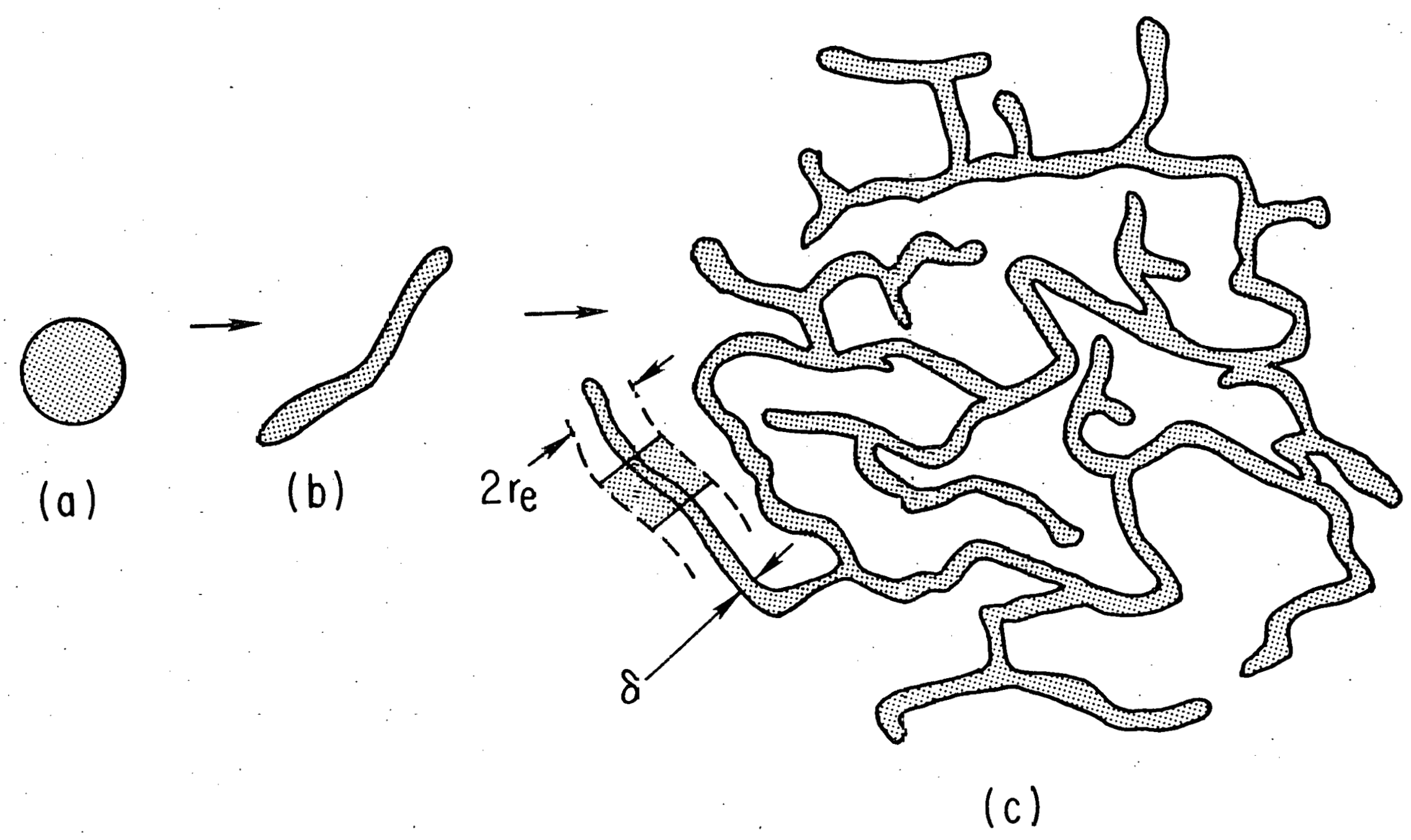

773886

Fig. 1. The Evolution of area mapping. 